\title{
Thermal diffusion effect of a regenerator with complex flow channels
}

\author{
S. Hasegawa, ${ }^{\mathrm{a},{ }^{*}}$ Y. Ashigaki, ${ }^{\mathrm{b}}$ Mariko Senga ${ }^{\mathrm{b}}$ \\ ${ }^{a}$ Department of Prime Mover Engineering, Tokai University, Kanagawa 259-1292, Japan \\ ${ }^{\mathrm{b}}$ Graduate school of Engineering, Tokai University, Kanagawa 259-1292, Japan
}

(Received XX XX 2014; accepted XX XX 2014; published

online XX XX 2014)

\begin{abstract}
The thermal diffusion effect caused by an oscillatory flow has a large effect on the thermal efficiency of a thermoacoustic engine. This study measures and compares the thermal diffusion effect caused by an oscillatory flow in regenerators with uniform and complex flow channels. In an experiment, a maximum heat flow originating from the thermal diffusion effect of $10.0 \mathrm{~W}$ is observed during the input of $0.1 \mathrm{~W}$ acoustic power in a regenerator with uniform flow channels at a temperature difference in the regenerator $\Delta T=100 \mathrm{~K} \cdot \operatorname{Im}\left[g_{D}\right]$, which is a parameter that governs the thermal diffusion effect, is found from the heat flow obtained in the experiment. Consequently, thermoacoustic theory for both regenerators with uniform and complex flow channels shows a good agreement. This result indicates that the regenerator with the random flow channels is equivalent to that with the uniform flow channels. Predicting the thermal diffusion effect according to the oscillatory flow is also possible.
\end{abstract} Copyright $\odot 2015$ Elsevier B.V. All rights reserved.

*Corresponding author. Email address: s.hasegawa @ tokai-u.jp 
List of Symbols

\begin{tabular}{|c|c|c|c|}
\hline $\bar{A}$ & cross-sectional area & $\overline{T_{C}}$ & ambient heat exchanger temperature \\
\hline$A_{\mathrm{Gas}}$ & cross-sectional areas of the gas & $T_{H}$ & hot heat exchanger temperature \\
\hline$A_{\text {Solid }}$ & $\begin{array}{l}\text { cross-sectional areas of the } \\
\text { regenerator }\end{array}$ & $\Delta T$ & $\begin{array}{l}\text { temperature difference between both } \\
\text { ends of the regenerator }\end{array}$ \\
\hline$c$ & adiabatic sound speed & $T_{m}$ & mean density in a duct \\
\hline$c_{p}$ & isobaric specific heat & $u$ & complex velocity amplitude \\
\hline$D$ & $\begin{array}{l}\text { flow path diameter of uniform } \\
\text { channel }\end{array}$ & $\left|\langle u\rangle_{R}\right|$ & $\begin{array}{l}\text { cross-sectional mean of the } \\
\text { oscillatory velocity in the regenerator }\end{array}$ \\
\hline$D_{h}$ & $\begin{array}{l}\text { hydraulic diameter of stacked } \\
\text { screen mesh }\end{array}$ & $V_{\text {gas }}$ & gas volume \\
\hline$d$ & wire diameter of the wire mesh & $V_{\text {holder }}$ & volume of the regenerator holder \\
\hline$f$ & frequency & $V_{\text {solid }}$ & $\begin{array}{l}\text { volume of the wires of the } \\
\text { stacked-screen regenerator }\end{array}$ \\
\hline$L$ & axial length of regenerators & $W$ & acoustic power \\
\hline$P$ & complex pressure amplitude & $Z$ & specific acoustic impedance \\
\hline$Q$ & heat flow & $\alpha$ & thermal diffusion coefficient \\
\hline$Q_{\text {prog }}$ & $\begin{array}{l}\text { heat flow caused by the traveling } \\
\text { wave component }\end{array}$ & $\delta$ & thermal penetration depth \\
\hline$Q_{\text {stand }}$ & $\begin{array}{l}\text { heat flow caused by the standing } \\
\text { wave component }\end{array}$ & $\varepsilon$ & porosity \\
\hline$Q_{D}$ & $\begin{array}{l}\text { heat flow caused by the thermal } \\
\text { diffusion effect }\end{array}$ & $\kappa$ & thermal conductivity \\
\hline$Q_{\kappa}$ & $\begin{array}{l}\text { heat flow caused by the simple } \\
\text { heat conduction }\end{array}$ & $v$ & viscosity coefficient \\
\hline$Q_{\text {Loss }}$ & heat leak & $\rho_{m}$ & mean density in a duct \\
\hline$r$ & flow channel radius & $\sigma$ & Prandtl number \\
\hline$r_{\text {eff }}$ & equivalent flow channel radius & $\varphi$ & $\begin{array}{l}\text { phase difference between pressure } \\
\text { and velocity }\end{array}$ \\
\hline$s$ & complex entropy amplitude & $\chi_{\alpha}, \chi_{v}$ & thermoacoustic functions \\
\hline$S_{g-s}$ & gas-solid contact surface area & $\omega$ & angular frequency, \\
\hline$T$ & $\begin{array}{l}\text { complex temperature amplitude of } \\
\text { the gas }\end{array}$ & & \\
\hline
\end{tabular}

\section{Introduction}

Thermoacoustic engines are basically built from pipes and a porous material with a temperature gradient (hereinafter referred to as the "regenerator"). Accordingly, these engines do not require moving parts, which have so far been indispensable in conventional thermal engines. In 1979, Ceperley theoretically posited that acoustic power is amplified when a traveling wave propagates in a regenerator with a temperature gradient [1]. In 1998, Yazaki et al. realized Ceperley's proposal as a loop-type thermoacoustic engine [2], which implies that regenerators are an important component in thermoacoustic engines. Thermoacoustic engine regenerators can be broadly classified into two categories. The first category comprises 
regenerators with uniform flow channels, including honeycomb ceramics with multiple square pores (Fig. 1), parallel plates, and pin arrays of bundled circular tubes. A regenerator with a flow channel radius smaller than the thermal penetration depth is necessary for a thermoacoustic engine to achieve high thermal efficiency [2]. However, manufacturing a regenerator with uniform flow channels, such as honeycomb ceramics, that produces small pores is difficult using the current technology. The second category comprises regenerators with complex flow channels. The equivalent to micro-pores can be comparatively and easily realized in regenerators with complex flow channels through stacking layers [3]. Figure 2 shows stacks of metal meshes. Reticulated vitreous carbon (RVC) [4], steel wool, and the like are used in regenerators with complex flow channels. In practice, stacks of metal meshes are used in regenerators for thermoacoustic engines with a high thermal efficiency [5,6]. The quantification of acoustic propagation and heat flow properties with respect to complex flow channels is an important task in the practical application of the thermoacoustic phenomenon. The acoustic propagation and heat flow properties within a regenerator with uniform flow channels can be understood according to the thermoacoustic wave equation by Rott [7].

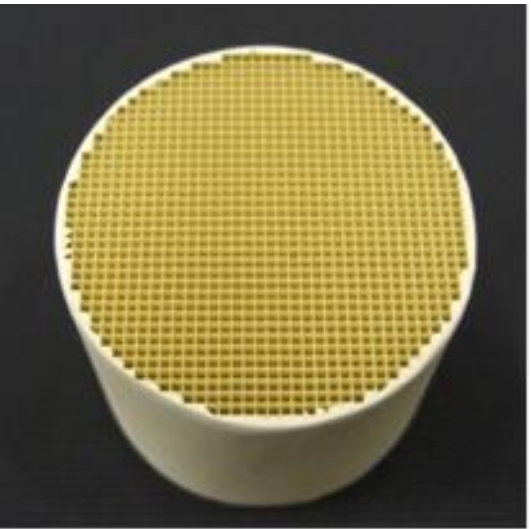

Fig. 1. Honeycomb ceramics used for regenerators $\left(2 r_{0}=1.18 \mathrm{~mm}\right.$ for the regenerator in the photograph). 


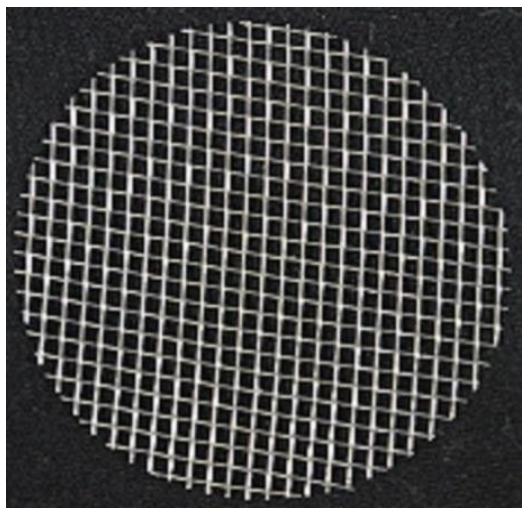

Fig. 2. Metal mesh used for regenerators (mesh \#16 for the mesh in the photograph).

Swift [8] and Tominaga [9] develop the thermoacoustic wave equation into thermoacoustic theory. Thermoacoustic theory is first applied to parallel plates and circular tubes. Arnott et al. developed thermoacoustic theory for equilateral triangular and rectangular flow channel geometries [10]. However, determining an accurate flow channel geometry for a regenerator with complex flow channels is difficult. For this reason, understanding the acoustic propagation and heat flow properties using thermoacoustic theory is usually difficult. It is necessary to empirically determine an equivalent flow channel diameter to understand the properties of a regenerator with complex flow channels. Wilen considered the RVC and found a thermoacoustic function for the acoustic propagation properties of a regenerator by measuring the amplitude from a speaker and the RVC internal and external pressures [11]. His comparisons with the analytical solutions for various flow channel geometries showed that this result was equivalent to that of a parallel plate. Furthermore, Ueda measured the pressure and velocity of a small-amplitude acoustic field around the inlet and outlet of a stacked-screen regenerator. He also compared the transfer matrix with that of a circular tube assembly [3], and found the following empirical formula for the equivalent flow channel radius $r_{\text {eff }}$ of a stacked-screen regenerator:

$$
r_{\text {eff }}=\frac{\sqrt{D_{h} d}}{2}
$$

Here, $D_{h}$ is the hydraulic diameter, and $d$ is the wire mesh diameter. Half the hydraulic diameter $\left(D_{h} / 2\right)$ is employed as the characteristic radius $r$ of a stacked-screen regenerator. $D_{h}$ is defined as four times the ratio of the gas volume $\left(V_{\text {gas }}\right)$ to the gas-solid contact surface area 
$\left(S_{g-s}\right)$ in a stacked-screen regenerator, that is,

$$
D_{h}=\frac{4 V_{\text {gas }}}{S_{\text {g-s }}} .
$$

$V_{\text {gas }}$ is estimated from the equation $V_{\text {gas }}=V_{\text {holder }}-V_{\text {solid, }}$, where $V_{\text {holder }}$ and $V_{\text {solid }}$ denote the volumes of the regenerator holder and wires of the stacked-screen regenerator, respectively. $S_{g-s}$ is estimated from the wire and inner-side surface areas of the regenerator holder. Much research on the regenerators with complex flow channels have been performed in this manner from the perspective of acoustic propagation properties. Meanwhile, research on the heat flow properties of a regenerator is less advanced. Here, there are two kinds of heat flow: one where heat is pumped from a low temperature to a high temperature using acoustic power, and another where heat is transported from a high temperature to a low temperature by the thermal diffusion effect. This thermal diffusion effect is called the dream pipe effect $[12,13,14]$, and Kruzweg and Zhao studied this effect with liquids as the subject [12]. The thermal diffusion effect is essential when using a thermoacoustic phenomenon in a heat pump or other heat transport devices. However, thermal efficiency greatly deteriorates in the case of using a thermoacoustic phenomenon in a thermoacoustic engine or thermoacoustic refrigerator. Accordingly, a quantitative understanding of heat flow in the regenerator is important. Heat flow $Q$ and acoustic power $W$ can be expressed herein as follows:

$$
\begin{aligned}
& Q=A \rho_{m} T_{m}\left\langle\langle s \cdot u\rangle_{t}\right\rangle_{R}=A \rho_{m} c_{p}\left\langle\langle T \cdot u\rangle_{t}\right\rangle_{R}-W, \\
& W=A\left\langle\langle P \cdot u\rangle_{t}\right\rangle_{R} .
\end{aligned}
$$

$\rho_{m}$ is the mean density in a duct; $T_{m}$ is the mean temperature; $s$ is the complex entropy amplitude; $c_{p}$ is the isobaric specific heat; $T$ is the complex temperature amplitude of the gas; $u$ is the complex velocity amplitude; $P$ is the complex pressure amplitude; \langle\rangle$_{t}$ represents the time average over a period; and \langle\rangle$_{R}$ represents the cross-section average. $Q$ can be determined if $P, T$, and $u$ are known. $P$ can be measured using a pressure transducer, while $u$ can be determined using a laser Doppler velocimeter or other measuring devices. However, little research has been conducted on the $T$ measurement. Huelsz and Ramos used a cold wire anemometer to measure the oscillatory temperature of uniform flow channels with respect to a standing wave and its phase with respect to the oscillatory pressure [15]. Their 
results showed a comparatively good agreement with Rott's linearized theory [7]. They also pointed out the necessity of accurately correcting the phase delay of the anemometer with respect to the oscillatory temperature [15]. Tashiro et al. used thermocouples to measure the radial distribution of the temperature in uniform flow channels [16]. The response function they obtained as a result agrees well with thermoacoustic theory. In this manner, no instances of directly measuring the heat flow in a regenerator have been reported although several measurement examples of $T$ within the uniform flow channels have been used. Accordingly, we measure the heat flow in a regenerator caused by an acoustic wave in a honeycomb ceramic with uniform flow channels [17]. From the results, we subsequently demonstrate that the equivalent heat capacity per unit area can be regulated by the dimensionless parameter $r / \delta$ $[8,18]$, which governs the acoustic wave propagation. Here, $r / \delta$ is a dimensionless parameter determined by the properties of the acoustic wave that propagates in the flow channel. This parameter is expressed by the flow channel radius $r$ and the thermal penetration depth $\delta$ of the flow channel. As above, the heat flow properties of a regenerator with uniform flow channels can be understood from the results of several previous studies. However, in order to obtain the micropores as described above, regenerators with complex flow channels are primarily used in thermoacoustic engines and thermoacoustic refrigerators. The heat flow quantification, with respect to complex flow channels, is an important task for realizing and implementing highly efficient thermoacoustic devices. Accordingly, we measure and compare the heat flow in a regenerator with uniform flow channels and a regenerator with complex flow channels. Directly measuring s and $T$ within the complex flow channels is difficult. Hence, we attempt to use thermoacoustic theory to isolate and measure the heat flow originating from the thermal diffusion effect $[12,17]$. The main factor causing a decrease in the efficiency of thermoacoustic engines and refrigerators is the heat flow caused by the thermal diffusion effect $[9,17]$ as described above. Therefore, a quantitative understanding of the heat flow caused by the thermal diffusion effect is necessary to improve the efficiency of thermoacoustic devices. This study proposes a method to separate the heat flow caused by the thermal diffusion effect from the experimental result based on thermoacoustic theory $[8,9]$. 
The heat flow in a regenerator with uniform flow channels is quantitatively compared with that in a regenerator with complex flow channels.

\section{Experimental apparatus}

We performed an experiment to measure the heat flow caused by an acoustic wave in a regenerator, with respect to both cases of a regenerator with uniform flow channels and that with complex flow channels. We also compared their properties. The honeycomb ceramic regenerator with a square flow channel geometry (Fig. 1) was prepared for the uniform flow channel regenerator. The stacked-screen mesh regenerator comprising a stack of stainless wire meshes (Fig. 2) was prepared for the complex channel regenerator. The axial length $L$ of both regenerators was $30 \mathrm{~mm}$. Table 1 lists the properties of the honeycomb ceramic regenerator. Table 2 presents the properties of the stacked-screen regenerator used in this experiment. The equivalent flow channel radius $r_{\text {eff }}$ of the stacked-screen regenerator was obtained using Eq. (3). Fig. 3 shows the experimental apparatus used for the flow measurements. The experimental apparatus consisted of a unit comprising opposed woofer speakers (FW108N, Fostex), stainless pipe with $40 \mathrm{~mm}$ inner diameter, copper heat exchanger, and regenerator. Fig. 4 shows a heat exchanger.

The gas contained inside was air at atmospheric pressure. The woofer speakers placed on both sides were driven by amplifying the signal from a signal generator (DG4062, Rigol) using a power amplifier (P1000S, YAMAHA). The amplitude and phase of the woofer speakers were adjusted using the signal generator in addition to the frequency to realize a low specific acoustic impedance of around $0.05 \rho_{m} c$ at the regenerator position. $\rho_{m} c$ was defined as the characteristic acoustic impedance of an acoustic wave propagating in free space. This variable was provided by the mean density of the gas $\rho_{m}$ and the adiabatic sound speed $c$. The heat exchanger had a fin shape with an axial length of $30 \mathrm{~mm}$, and was made from copper arranged in parallel with a plate interval of $2 \mathrm{~mm}$. A sheath heater for the hot heat exchanger (HHX) was regulated using a DC power supply to make the temperature difference $\Delta T$ between both regenerators be $100 \mathrm{~K}$ and maintain a temperature of $T_{H}=403 \mathrm{~K}$. The ambient 
heat exchanger was maintained at $T_{C}=303 \mathrm{~K}$ by circulating cooling water. The semiconductor pressure transducers for the air pressure (PD104K, JTEKT) were attached in three places to the wall surface of the stainless pipes affixed to the unit. The acoustic field in the tube was measured using a Two-sensor method [19]. The regenerator was placed at a position $1.3 \mathrm{~m}$ from each of the woofer speakers at both pipe ends.

Table 1. Geometrical properties of ceramic honeycombs. $D$ denotes the flow path diameter.

\begin{tabular}{cccc}
\hline \hline Type & HA & HB & HC \\
\hline$D=2 r(\mathrm{~mm})$ & 0.67 & 1.18 & 2.11 \\
Porosity $\varepsilon(\%)$ & 83.3 & 86.3 & 69.0 \\
\hline \hline
\end{tabular}

Table 2. Geometrical properties of the stacked-screen regenerators. $D_{h}$ denotes the hydraulic diameter.

\begin{tabular}{llll}
\hline \hline \multicolumn{1}{c}{ Type } & RA & RB & RC \\
\hline Mesh No. & 16 & 10 & 6 \\
Wire diameter $d(\mathrm{~mm})$ & 0.2 & 0.4 & 0.6 \\
Porosity $\varepsilon(\%)$ & 76.4 & 71 & 73.6 \\
$D_{h}=2 r(\mathrm{~mm})$ & 2.16 & 5.57 & 9.26 \\
$r_{\text {eff }}(\mathrm{mm})$ & 0.328 & 0.571 & 0.914 \\
\hline \hline
\end{tabular}

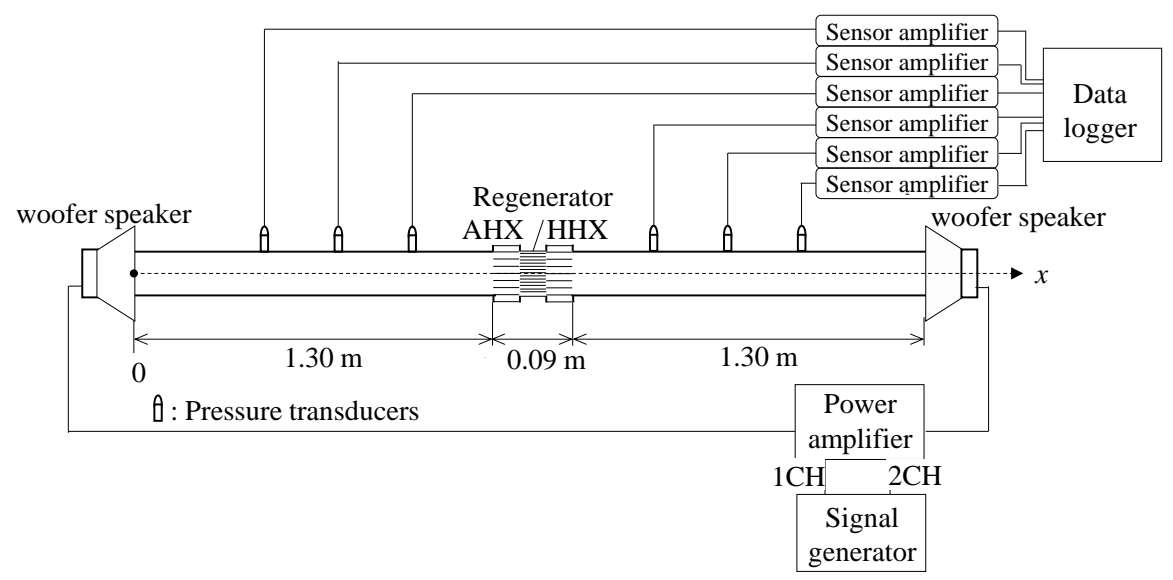


Fig. 3. Experimental apparatus (AHX: ambient heat exchanger; HHX: hot heat exchanger).

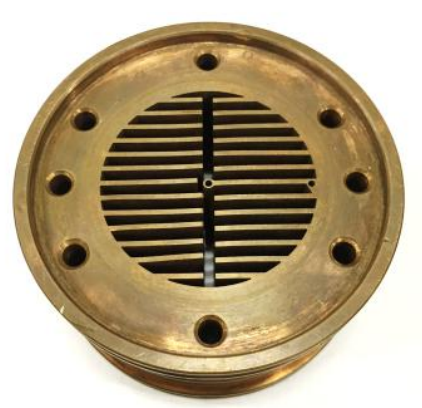

Fig. 4. Heat exchanger (ambient and hot heat exchangers have the same shape).

\section{Heat flow isolation method}

As shown in Eq. (3), measuring $s$ and $T$ was necessary to directly measure the heat flow $Q$. However, directly measuring $s$ and $T$ in the complex flow channels was difficult. The main factor that decreased the efficiency of a thermoacoustic engine or refrigerator was the thermal diffusion effect $[9,17]$. Accordingly, only the heat flow originating from the thermal diffusion effect was isolated from the experimental results based on thermoacoustic theory. Heat flow occurred in conjunction with the gain/attenuation of acoustic power when an acoustic wave propagated in a regenerator with a temperature gradient. The $Q$ generated at this time can be expressed by Eq. (5) as follows according to thermoacoustic theory [9]:

$$
Q=Q_{\text {prog }}+Q_{\text {stand }}+Q_{\mathrm{D}}+Q_{\kappa}+Q_{\text {Loss }} \text {. }
$$

where $Q_{\text {prog }}$ is the heat flow caused by the traveling wave component; $Q_{\text {stand }}$ is the heat flow caused by the standing wave component; $Q_{D}$ is the heat flow caused by the thermal diffusion effect; $Q_{\kappa}$ is the heat flow caused by the simple heat conduction; and $Q_{\text {Loss }}$ is the heat leak, such as atmospheric heat dissipation. The contribution of each component was difficult to measure. Therefore, $Q_{D}$ was isolated according to the experimental conditions. The components of each heat flow are expressed by the following equations [9]:

$$
Q_{\text {prog }}=-\frac{A_{\mathrm{Gas}} \operatorname{Re}[g] \operatorname{Re}[Z]}{2}\left|\langle u\rangle_{R}\right|^{2} \cos \varphi,
$$




$$
Q_{\text {stand }}=-\frac{A_{\text {Gas }} \operatorname{Im}[g] \operatorname{Re}[Z]}{2}\left|\langle u\rangle_{R}\right|^{2} \sin \varphi,
$$

$$
Q_{\mathrm{D}}=\frac{A_{\mathrm{Gas}} \rho_{m} c_{p}}{2 \omega} \operatorname{Im}\left[g_{D}\right]\left|\langle u\rangle_{R}\right|^{2} \frac{\Delta T}{L},
$$$$
Q_{\kappa}=-A_{\mathrm{Solid}} \kappa \frac{\Delta T}{L},
$$

where $A_{\text {Gas }}$ and $A_{\text {Solid }}$ are the cross-sectional areas of the gas and the regenerator; $\varphi$ is the phase difference between pressure and velocity; $\omega$ is the angular frequency; $\kappa$ is the thermal conductivity; and $\Delta T$ is the temperature difference between both regenerator ends. $g, g_{D}$, and the specific acoustic impedance $Z$ are expressed as follows:

$$
\begin{aligned}
& g=\frac{\chi_{\alpha}-\breve{\chi}_{v}}{(1+\sigma)\left(1-\breve{\chi}_{v}\right)}, \\
& g_{D}=\frac{\chi_{\alpha}-\sigma \chi_{\alpha}}{\left|1-\chi_{v}\right|^{2}\left(1-\sigma^{2}\right)}, \\
& Z=\frac{p}{\langle u\rangle_{R}},
\end{aligned}
$$

where $\sigma$ is the Prandtl number calculated as 0.71 because the atmospheric pressure air is the working gas. In addition, $\chi_{\alpha}$ and $\chi_{v}$ are thermoacoustic functions $[9,20]$ that depend on $r / \delta$ [8]. $\chi_{\alpha}$ and $\chi_{v}$ are written as follows if the flow channel has circular cross sections:

$$
\chi_{\alpha, v}=\frac{2 J_{1}\left\{(j-1)\left(r / \delta_{\alpha, v}\right)\right\}}{(j-1)\left(r / \delta_{\alpha, v}\right) J_{0}\left\{(j-1)\left(r / \delta_{\alpha, v}\right)\right\}},
$$

where $J_{1}$ and $J_{0}$ are the first- and zeroth-order Bessel functions, respectively; $v$ is the viscosity coefficient, and $\alpha$ is the thermal diffusion coefficient. Accordingly, $r / \delta$ is calculated using $r$ or $r_{\text {eff }}$ and the mean temperature of the regenerator in the axial direction is $\left(=\left(T_{H}+T_{C}\right) / 2\right)$, and $\delta_{\alpha, v}=\sqrt{2(\alpha, v) / \omega}$ here.

We first measured $Q_{\kappa}$ and $Q_{\text {Loss }}$, which were the input electrical power necessary to maintain a regenerator temperature difference $\Delta T=100 \mathrm{~K}\left(T_{H}=403 \mathrm{~K}, T_{C}=303 \mathrm{~K}\right)$ when no acoustic wave was introduced. The $Q_{\kappa}$ for the regenerator used in the experiment was about 1.72-3.89. The flow at that time corresponds to Eq. (5). Therefore, the heat flow according to the forced oscillation $Q_{\text {acoustic }}$ can be estimated by deducting $Q_{\kappa}$ and $Q_{\text {Loss }}$ from this to obtain the following formula:

$$
Q_{\text {acoustic }}=Q_{\text {prog }}+Q_{\text {stand }}+Q_{\mathrm{D}}
$$


The acoustic wave, when it was input, was adjusted using the woofer speakers placed at both ends and the signal generator, such that $\varphi$ was $0^{\circ}$ and $Z$ was around $0.05 \rho_{m} c$ at the regenerator position. First, the heat flow caused by the standing wave $Q_{\text {stand }}$ can be ignored by making the phase difference between pressure and velocity $\varphi=0^{\circ}$ :

$$
Q_{\text {acoustic }}=Q_{\text {prog }}+Q_{\mathrm{D}} \text {. }
$$

We then considered $\left|Q_{D} / Q_{\text {prog }}\right| \cdot \cos \varphi=1$ because $\varphi$ was $0^{\circ}$. The following formula was then obtained:

$$
\left|\frac{Q_{\mathrm{D}}}{Q_{\mathrm{prog}}}\right|=\frac{c_{p} \Delta T}{0.05 \omega c L}\left|\frac{\operatorname{Im}\left[g_{D}\right]}{\operatorname{Re}[g]}\right| .
$$

Here, the same parameters as in the experimental conditions were input into Eq. (6). Furthermore, $r / \delta$ was varied from 0.1 to 10.0 . However, the maximum frequency of $190 \mathrm{~Hz}$ was substituted for $\omega$ in the experimental conditions mentioned below. Fig. 5 shows the calculation results, where the $Q_{D}$ magnitude was in excess of 10-500 times $Q_{\text {prog }}$ for the $r / \delta$ range, in which the experiment was performed (detailed experiment conditions were given below, but $r / \delta=0.6-7)$. Accordingly, $Q_{\text {prog }}$ became relatively insignificant by decreasing with the acoustic interference:

$$
Q_{\text {acoustic }} \cong Q_{\mathrm{D}} \text {. }
$$

Isolating the flow caused by the acoustic wave as $Q_{D}$ and measuring the thermal diffusion effect caused by the acoustic wave were possible by using the above mentioned isolation method.

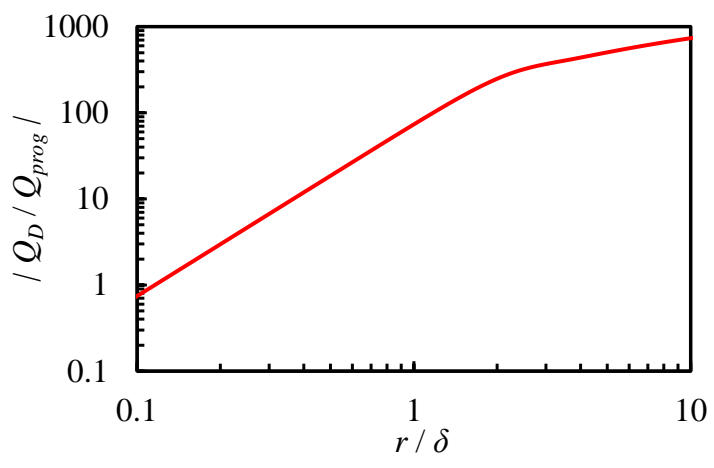

Fig. 5. Relation between $r / \delta$ and $\left|Q_{D} / Q_{\text {prog }}\right|$. 


\section{Experimental results}

In this study, we performed an experiment to measure the heat flow caused by an acoustic wave for both regenerators with uniform and complex flow channels. The frequency $f$ of the woofer speakers was set at $45,65,85,110,140$, and $190 \mathrm{~Hz} . Q_{\mathrm{D}}$ for each frequency was measured, and the cross-sectional mean of the oscillatory velocity in the regenerator $\left|\langle u\rangle_{R}\right|$ was adjusted to $2 \mathrm{~m} / \mathrm{s}$. $\left|\langle u\rangle_{R}\right|$ was determined by averaging that value $\left|\left\langle u_{1}\right\rangle_{R}\right|,\left|\left\langle u_{2}\right\rangle_{R}\right|$ and dividing by the regenerator porosity $\varepsilon$. Where, $\left|\left\langle u_{1}\right\rangle_{R}\right|,\left|\left\langle u_{2}\right\rangle_{R}\right|$ were in the position connecting the duct at the ambient (AHX) and HHX by using the Two-sensor method [19] and the measured pressure oscillation.

Figure 6 shows the relationship between the estimated $Q_{\mathrm{D}}$ in the regenerator and $r / \delta$. Panel (a) denoted the results for the honeycomb ceramic regenerator, whereas panel (b) presented those for the stacked-screen regenerator. We found that $Q_{\mathrm{D}}$ was large when $r / \delta$ was low for both regenerators (panels (a) and (b)) because the axial displacement amplitude was large. A maximum $Q_{\mathrm{D}}$ of $10.0 \mathrm{~W}$ was observed when the regenerator with uniform flow channels was used. The acoustic power input to the AHX at that time was $0.1 \mathrm{~W}$. The acoustic power was found by multiplying the oscillatory pressure measured by the semiconductor pressure transducers by the oscillatory velocity found using the Two-sensor method [19]. The abovementioned result indicated that the thermal diffusion effect caused by the oscillatory flow largely affected the thermal efficiency of the thermoacoustic engine.

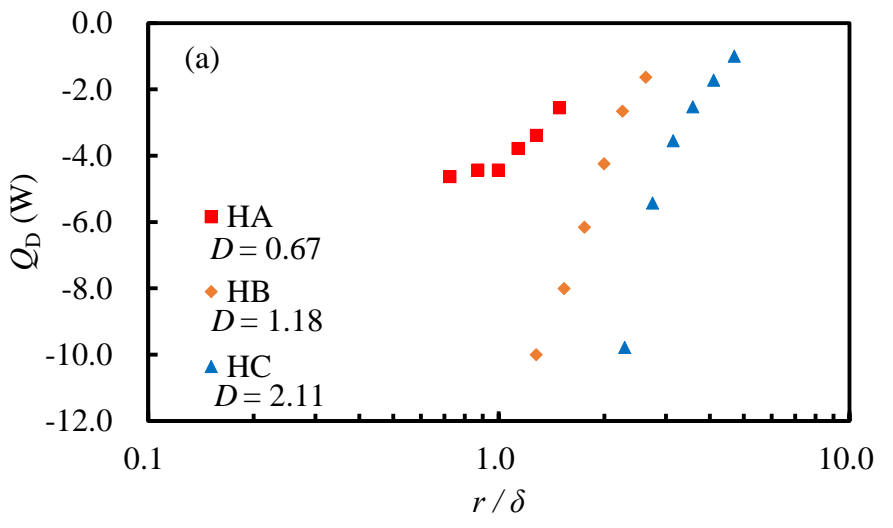




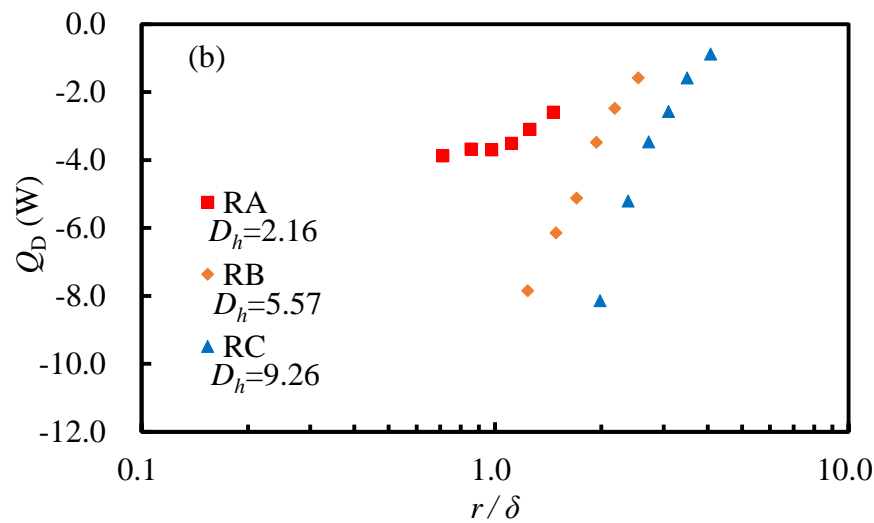

Fig. 6. Relation between $r / \delta$ and the measured heat flow by oscillatory flow $Q_{\mathrm{D}}$ : (a) honeycomb ceramic regenerator with uniform flow channels and (b) stacked-screen regenerator with complex flow channels.

We then estimated the dimensionless parameter $\operatorname{Im}\left[g_{D}\right]$ that governed $Q_{D}$ from the measured value. We investigated the relationship of $\operatorname{Im}\left[g_{D}\right]$ with $r / \delta$ for both the honeycomb ceramic and stacked-screen regenerators. $\operatorname{Im}\left[g_{D}\right]$ was found from the results below by using Eq. (8) and the measured $Q_{\mathrm{D}}$.

$$
\operatorname{Im}\left[g_{D}\right]=\frac{2 \omega L A_{\text {Gas }}}{c_{p} \rho_{m} \Delta T\left|\langle u\rangle_{R}\right|^{2}} Q_{D} .
$$

Moreover, $\operatorname{Im}\left[g_{D}\right]$ was found by theoretically using Eq. (11). Fig. 7 shows the results. Panel (a) denoted the results for the honeycomb ceramic regenerator, whereas panel (b) showed those for the stacked-screen regenerator. The plotted points and line in the diagram showed the measured and theoretical values, respectively. We first compared the theoretical with the measured values. The theoretical and experimental values for $\operatorname{Im}\left[g_{D}\right]$, which governed the heat flow, were in agreement in the entire region in both panels (a) and (b), although different frequencies and flow channel diameters were used in the experiment. This result indicated that the dimensionless parameter $r / \delta$ that governed the acoustic wave propagation in the flow channel was also a governing factor with respect to heat transport caused by the acoustic wave. $\operatorname{Im}\left[g_{D}\right]$ was the largest in the $1<r / \delta<3$ range. Hence, the $Q_{\mathrm{D}}$ measured in this study was essentially caused by an irreversible process. Ueda's Eq. (3) was used to find the equivalent flow channel radius $r_{\text {eff }}$ of the stacked-screen regenerator. The result, where $Q_{\mathrm{D}}$ in the regenerator can be predicted, was obtained within the scope of the experimental 
conditions according to Eq. (7) in the same manner as that in the uniform flow channels. This result indicated that a regenerator with complex flow channels can be considered equivalent to one with uniform flow channels. Furthermore, the thermal diffusion effect caused by the oscillatory flow can be predicted.
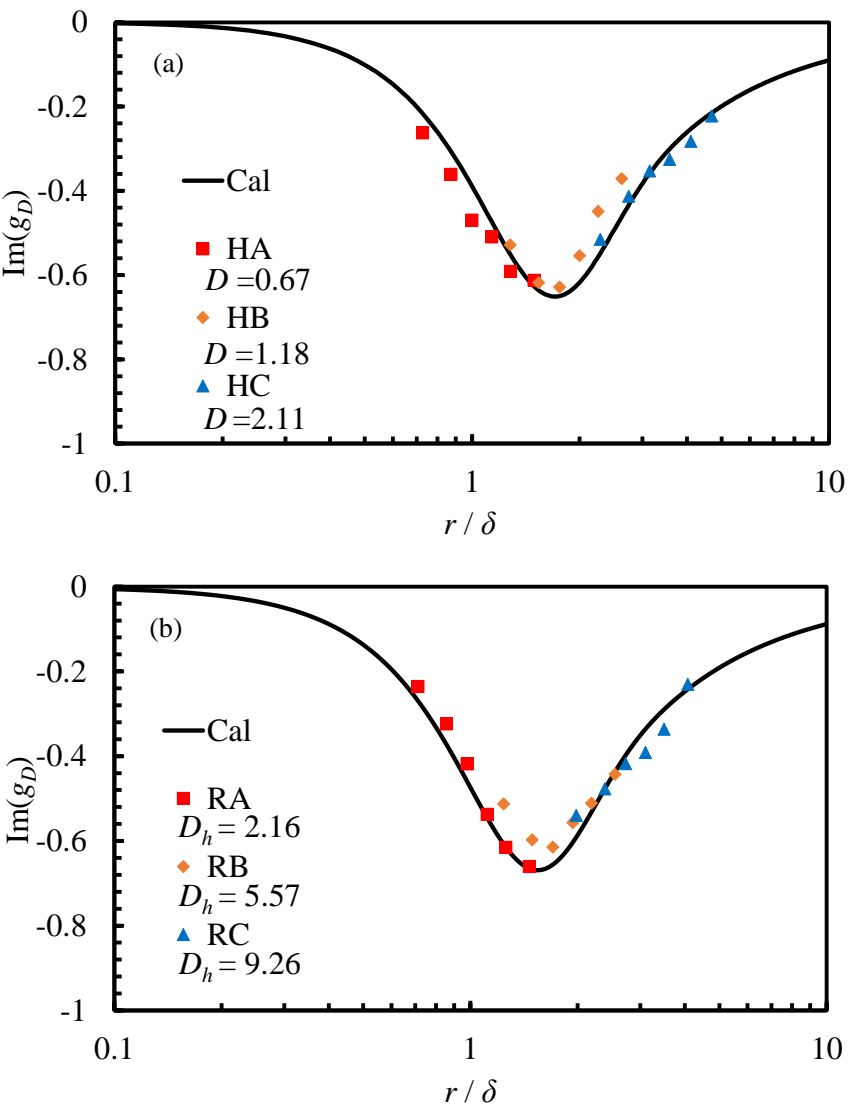

Fig. 7. Relation between $r / \delta$ and $\operatorname{Im}\left[g_{D}\right]$ : (a) honeycomb ceramic regenerator with uniform flow channels and (b) stacked-screen regenerator with complex flow channels. 


\section{Conclusion}

In this study, we measured the heat flow caused by the thermal diffusion effect when using honeycomb ceramic and stacked-screen regenerators. In the experiment, the maximum heat flow originating from the thermal diffusion effect of $10.0 \mathrm{~W}$ was observed during the input of $0.1 \mathrm{~W}$ acoustic power in the regenerator with uniform flow channels. The result indicated that the thermal diffusion effect caused by the oscillatory flow largely affected the thermal efficiency of the thermoacoustic engine. Parameter $\operatorname{Im}\left[g_{D}\right]$, which governed the thermal diffusion effect, was found from the heat flow obtained in the experiment. Consequently, both the honeycomb ceramic and stacked-screen regenerators showed good agreement with thermoacoustic theory. This finding indicated that a regenerator with complex flow channels can be considered equivalent to one with uniform flow channels. Furthermore, predicting the thermal diffusion effect according to the oscillatory flow was possible. These results helped easily predict the heat flow in a regenerator containing complex flow channels, and can be applied in designing an actual thermoacoustic engine.

\section{Acknowledgments}

This study was financially supported by the Advanced Low Carbon Technology Research and Development Program (ALCA) in 2015 (grant no. 13414425) from the Japan Science and Technology Agency.

\section{References}

[1] P.H. Ceperley, A pistonless Stirling engine - the traveling wave heat engine, The Journal of the Acoustical Society of America 66 (1979) 1508-1513.

[2] T. Yazaki et al., Traveling wave thermoacoustic engine in a looped tube, Physical Review Letters 81 (1998) 3128-3131.

[3] Y. Ueda, Experimental evaluation of the acoustic properties of stacked-screen regenerators, The Journal of the Acoustical Society of America 125 (2009) 780-786.

[4] Reticulated vitreous carbon (RVC) is manufactured by Energy Research and Generation, 
Inc., 900 Stanford Avenue, CA 94608.

[5] S. Backhaus, G.W. Swift, A thermoacoustic Stirling heat engine, Nature 399 (1999) $335-338$

[6] M.E.H. Tijani, S. Spoelstra, A high performance thermoacoustic engine, Journal of Applied Physics 110 (2011) 093519-1-6.

[7] N. Rott, Thermoacoustics, Advances in Applied Mechanics 20 (1980) 135-17

[8] G. W. Swift, Thermoacoustics: A Unifying Perspective for Some Engines and Refrigerators (Acoustical Society of America through the American Institute of Physics, Melville, New York, (2002).

[9] A. Tominaga, Thermodynamic aspects of thermoacoustic theory, Cryogenics 35 (1995) $427-440$.

[10] W.P. Arnott et al., General formulation of thermoacoustics for stacks having arbitrarily shaped pore cross sections, The Journal of the Acoustical Society of America 90 (1991) 3228-3237.

[11] L.A. Wilen, Dynamic measurements of the thermal dissipation function of reticulated vitreous carbon, The Journal of the Acoustical Society of America 109 (2000) 179-184.

[12] U.H. Kurzweg, L. Zhao, Heat transfer by high-frequency oscillations - a new hydrodynamic technique for achieving large effective thermal conductivities, Physics of Fluids 27 (1984) 2624-2627.

[13] U.H. Kurzweg, Enhanced Heat Conduction in Fluids Subjected to Sinusoidal Oscillations, Journal of Heat Transfer 107 (1985), 459-462.

[14] S. Nishio, X. Shi, W. Zhang, Oscillation-induced heat transport: heat transport characteristics along liquid-columns of oscillation-controlled heat transport tubes, International Journal of Heat and Mass Transfer 38 (1995) 2457-2470.

[15] G. Huelsz, E. Ramos, Temperature measurements inside the oscillatory boundary layer produced by acoustic waves, The Journal of the Acoustical Society of America 103 (1998) 1532-1537.

[16] Y. Tashiro et al., Calibration of a thermocouple for measurement of oscillatory 

temperature, Review of Scientific Instruments 76 (2005) 124901-1-5.

[17] S. Hasegawa, K. Kondo, Y. Oshinoya, Experimental verification of heat transport by acoustic wave, Applied Thermal Engineering 78 (2015) 551-555.

[18] T. Yazaki, Y. Tashiro, T. Biwa, Measurements of sound propagation in narrow tubes, Proceedings of the Royal Society of London A 463 (2007) 2855-2862.

[19] A. Fusco, W. Ward, G. Swift, Two-sensor power measurements in lossy ducts, The Journal of the Acoustical Society of America 91 (1992) 2229-2235.

[20] N. Rott, Damped and thermally driven acoustic oscillations, Journal of Applied Mathematics and Physics 20 (1969) 230-243. 